\title{
Effects of L-theanine or caffeine intake on changes in blood pressure under physical and psychological stresses
}

Ai Yoto*, Mao Motoki, Sato Murao and Hidehiko Yokogoshi

\begin{abstract}
Background: L-theanine, an amino acid contained in green tea leaves, is known to block the binding of L-glutamic acid to glutamate receptors in the brain, and has been considered to cause anti-stress effects by inhibiting cortical neuron excitation. Both L-theanine and caffeine, which green tea contains, have been highlighted for their beneficial effects on cognition and mood.

Methods: In this study, we investigated the effects of orally administered L-theanine or caffeine on mental task performance and physiological activities under conditions of physical or psychological stress in humans. Fourteen participants each underwent three separate trials, in which they orally took either L-theanine + placebo, caffeine + placebo, or placebo only.

Results: The results after the mental tasks showed that L-theanine significantly inhibited the blood-pressure increases in a high-response group, which consisted of participants whose blood pressure increased more than average by a performance of a mental task after placebo intake. Caffeine tended to have a similar but smaller inhibition of the blood-pressure increases caused by the mental tasks. The result of the Profile of Mood States after the mental tasks also showed that L-theanine reduced the Tension-Anxiety scores as compared with placebo intake.
\end{abstract}

Conclusions: The findings above denote that L-theanine not only reduces anxiety but also attenuates the blood-pressure increase in high-stress-response adults.

Keywords: L-theanine, Caffeine, Blood pressure, Acute stress, Profile of mood states

\section{Background}

To live a healthier life in so-called high-stress modern society, a growing interest in natural, minimally processed, nutritional, and healthy foods is spreading around the world, and many kinds of functional food ingredients have recently become widely used due to their health benefits. L-theanine became one of those popular items since its multiple roles in the central and autonomic nervous systems received attention. Animal studies have revealed that L-theanine affected dopamine and serotonin concentrations in the brain, underlying its anxiolytic effect [1,2]. Several reports have found

\footnotetext{
* Correspondence: ai_yoto@hotmail.com

Laboratory of Nutritional Biochemistry, School of Food and Nutritional Sciences, University of Shizuoka, 52-1 Yada, Suruga-ku, Shizuoka 422-8526, Japan
} theanine administration, indicating that L-theanine could lead to a relaxed and alert state [3,4]. Kimura (2007) reported that L-theanine intake reduced heart rate and salivary immunoglobulin $\mathrm{A}$ responses to an acute stress task (an arithmetic task), suggesting that Ltheanine could reduce stress by inhibiting cortical neuron excitation [5]. Moreover, animal studies have found that L-theanine reduced blood pressure in hypertensive rats [6,7]. It is known that stress can elevate blood pressure by stimulating the nervous system to produce large amounts of vasoconstricting hormones that increase blood pressure [8,9], L-theanine may have inhibited the increase in blood pressure through its antistress effects on the autonomic nervous system. From these findings, it can be hypothesized that L-theanine 
attenuates the stress responses in the autonomic nervous system induced by both physically and psychologically stressful tasks.

Caffeine, another major component of green tea, also has behavioral effects on autonomic nervous activities, and these effects are thought to be the opposite those of L-theanine. Caffeine is a CNS-stimulating drug that acts as an adenosine receptor antagonist in the brain $[10,11]$. Adenosine antagonism has been implicated as a contributor to the direct cardio-acceleratory effect of caffeine, which also increased blood pressure and respiration rate [12]. On the other hand, both caffeine and L-theanine were recently found to have beneficial effects on cognition and mood [13-15], but no study has compared these two components under conditions in which acute psychological and physical stresses increase blood pressure.

In this study, we investigated the effects of L-theanine or caffeine on mental task performance and the change in blood pressure caused by mental tasks as psychological stress and by the cold pressor test as physical stress.

\section{Methods}

The experiment conducted in this study was approved by the research ethics committee of the University of Shizuoka, and was carried out in accordance with the Declaration of Helsinki.

\section{Participants}

Sixteen healthy volunteers (students, eight men, eight women; ages, $22.8 \pm 2.1$ years) participated in the experiment individually at similar times of the day at an interval of 7 days. The data from two women were excluded from the analyses because they were absent on at least 2 experiment days owing to temporary illness. All participants were requested to avoid eating or drinking, except for water, from $3 \mathrm{~h}$ before the start of each trial.

\section{Treatment}

A cross-over, randomized, placebo-controlled design was used in this study. In total, three separate trials were performed, in which the participants orally took either Ltheanine (200 mg, Taiyo Kagaku Co., Tokyo, Japan) + placebo, caffeine (100 mg, Shiratori Pharmaceutical Co., Chiba, Japan) + placebo, or placebo only on each day. Dextrin (Nisshin Pharma Inc., Tokyo, Japan) was used as the placebo. All sample capsules were taken with 250 $\mathrm{mL}$ warm water at about $25^{\circ} \mathrm{C}$. Treatments were allocated using a Latin square design such that the order of treatments was counterbalanced across participants.

Yokogoshi et al. (1998) reported that L-theanine increased by $1 \mathrm{~h}$ at the latest in the serum, the liver, and the brain after administration, and thereafter decreased sharply in the serum and liver $[1,16]$. Van der Pijl et al. (2010) reported that L-theanine plasma concentration reached the peak between 32 and $50 \mathrm{~min}$ after oral ingestion, and its half-life ranged from $58 \mathrm{~min}$ to $74 \mathrm{~min}$ in humans [17]. Terashima et al. (1999). also reported that L-theanine could influence the secretion and function of neurotransmitters in the central nervous system even at $30 \mathrm{~min}$ after oral administration [18]. On the other hand, caffeine absorption from the gastrointestinal tract is rapid and reaches $99 \%$ in about $45 \mathrm{~min}$ after ingestion, while peak plasma caffeine concentration is reached between $15 \mathrm{~min}$ and $120 \mathrm{~min}$, and half-life ranges from $2.5 \mathrm{~h}$ to $4.5 \mathrm{~h}$ after oral ingestion in humans [19]. To allow a peak of both L-theanine and caffeine appears during the stress load period, sample treatment was decided to be taken at 36 min before the end of the mental tasks session (DT and AT as defined below), followed by subjective assessment which was performed from 38 min to $43 \mathrm{~min}$, physiological measurement from $44 \mathrm{~min}$ to $45 \mathrm{~min}$, and physical stress task session (CPT) from $45 \mathrm{~min}$ to $49 \mathrm{~min}$ after the sample treatment.

\section{Stress load task}

After each sample was taken, an auditory oddball target detection task (DT) lasting for $5 \mathrm{~min}$ each and an arithmetic mental task (AT) lasting for $10 \mathrm{~min}$ each were both imposed twice as the psychological stress load. In the DT, participants were required to click the left button of a computer mouse as quickly as possible to target stimuli (a single tone of $2,000 \mathrm{~Hz}$ lasting for $0.1 \mathrm{~s}$ ) that occur infrequently and irregularly within a series of standard stimuli (a single tone of $1,000 \mathrm{~Hz}$ lasting for $0.1 \mathrm{~s})$. The AT required participants to add two numbers (each from 1 to 9) that were being displayed on a PC monitor and to enter the answer through the keyboard quickly and accurately. The number and accuracy of the answers to the second AT, which was taken from $26 \mathrm{~min}$ to $36 \mathrm{~min}$ after each sample intake, were used for data analysis.

A cold pressor test (CPT) was taken to establish physical acute stress [20]. Participants were asked to immerse their right hand, past the level of the wrist, for $1 \mathrm{~min}$ in a bucket filled with slushy ice water $(1.5 \pm 0.3 \mathrm{C})$ and then to place the hand on the table nearby with a towel underneath the hand.

\section{Subjective assessment}

The Profile of Mood States (POMS) and the visual analogue scales (VAS) for subjective ratings on mood state were also completed before the intake as a basic control and after all of the mental tasks were finished.

The short version of POMS was used to assess distinct affective mood states. POMS is a popular tool that is widely used among psychologists and scientists in many 
fields. Six identifiable mood or affective states can be measured and were used for analysis in this study: Tension-Anxiety (T-A), Depression-Dejection (D), Anger-Hostility (A-H), Vigor-Activity (V), FatigueInertia (F), and Confusion-Bewilderment (C).

VAS comprised five scales including feelings of fatigue, relaxation, arousal, pressure, and tension. At the end of each trial, the subjects used the scales to rate their painful feelings about accomplishing the CPT and their feelings of annoyance about DT and AT.

\section{Physiological measurement}

Arterial pressure in each participant's left thumb was recorded continuously by Finometer Pro (FMS, Finapres Measurement Systems, Arnhem, the Netherlands). Simultaneously, skin temperature of the back of the left hand was recorded using a BioAmplifier (Polymate AP1132, TEAC, Tokyo, Japan). The sampling rate was $200 \mathrm{~Hz}$. As baseline data, both the blood pressure and skin temperature were measured for $1 \mathrm{~min}$ before the intake. Measurement after mental tasks (AMT) was also made for $1 \mathrm{~min}$ at $44 \mathrm{~min}$ after the intake of each sample, followed by measurement for $4 \mathrm{~min}$ after CPT was started.

Baseline data were calculated by averaging the $1 \mathrm{~min}$ data before each intake. Differences in blood pressure and skin temperature from the baseline were calculated using the mean value of every 10-s epoch for the above measurements after intake. The first 10-s epoch of the AMT was described as AMT1, and the second, third, fourth, fifth, and sixth 10-s epochs were described as AMT2, AMT3, AMT4, AMT5, and AMT6, respectively. Similarly, CPT1 to CPT6 for the CPT epochs, and RP1 to RP18 for epochs during the 3-min recovering period after the $1 \mathrm{~min}$ CPT were named respectively and used for the analysis.

\section{Procedure}

Figure 1 shows the experimental procedure. Each participant was required to attend a total of 3 study days, which were conducted 7 days apart, to ensure a sufficient washout between conditions. Prior to the start of the experiment, all participants were given the opportunity to familiarize themselves with all of the stress load tasks. The experiments took place in a quiet room. The room temperature was $26.4 \pm 1.1^{\circ} \mathrm{C}$, and the humidity was $51.5 \pm 6.8 \%$. On each experiment day, each participant entered the room and rested for $15 \mathrm{~min}$. During the resting time, a skin-surface temperature probe was attached, and POMS and VAS were completed. After the rest, a 1-min physiological measurement session to obtain baseline data took place, followed by sample treatment. After the oral administration, mental tasks were performed: DT (5 min), rest (2 $\mathrm{min})$, AT (10 $\mathrm{min})$, and

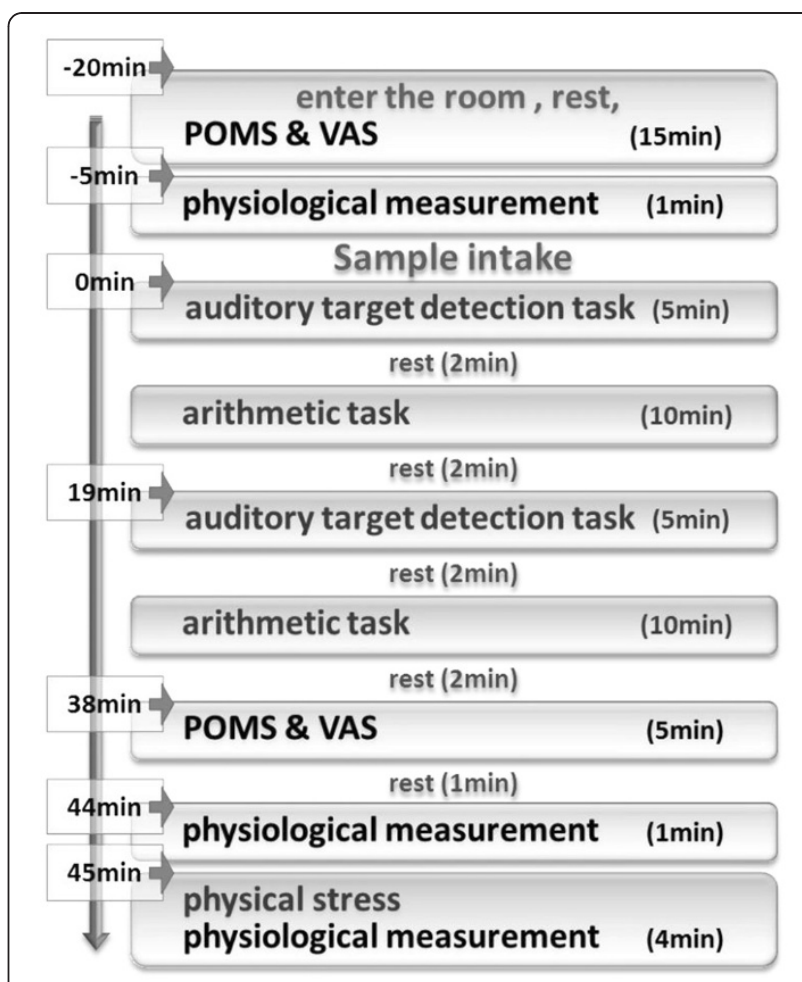

Figure 1 The experimental procedure.

rest ( $2 \mathrm{~min})$; the cycle was then repeated. Then, POMS and VAS and another 1-min measurement were completed again to obtain data after the mental tasks. CPT for $1 \mathrm{~min}$ was then started. At the same time, measurement was recorded for $4 \mathrm{~min}$ ( $1 \mathrm{~min}$ for CPT, $3 \mathrm{~min}$ for $\mathrm{RP}$ after CPT). At last, VAS about feelings of DT, AT, and CPT was completed.

\section{Statistical analysis}

Data were analyzed using IBM SPSS Statistics version 19. Prior to the primary statistical analysis, separate, one-way, repeated measures ANOVAs of the baseline data were conducted to ascertain any chance baseline differences across study days prior to the treatments.

L-theanine reduced blood pressure in spontaneously hypertensive rats but not in rats with normal blood pressure [6,7]. Thus it is considerable that L-theanine might act in different ways between people in whom stress increases whose blood pressure in different ways. With this in mind, we divided the participants into two groups after the experiment according to their changes in systolic blood pressure after the mental tasks in the placebo intake condition. The half of participants who showed greater than average changes in blood pressure were sorted into a high-response group and the other half into a low-response group.

Differences in blood pressure and skin temperature from the basic control were calculated and used for a 
Table 1 Changes in systolic blood pressure and diastolic blood pressure

Systolic blood pressure changes $(\mathrm{mmHg})$

\section{High-response group}

\section{Mean blood pressure}

SEM

Placebo Caffeine L-theanine Plac
Low-response group

Mean blood pressure

SEM

\begin{tabular}{|c|c|c|c|c|c|c|c|c|c|c|c|c|}
\hline Base & 0 & 0 & 0 & 0 & 0 & 0 & 0 & 0 & 0 & 0 & 0 & 0 \\
\hline AMT1 & 4.83 & 7.17 & 12.17 & 5.75 & 4.85 & 3.58 & 10.32 & 5.78 & 6.82 & 3.02 & 7.25 & 3.12 \\
\hline AMT2 & 3.05 & 5.55 & 13.82 & 3.48 & 6.04 & 3.48 & 5.62 & 2.07 & 1.36 & 3.84 & 5.23 & 2.06 \\
\hline AMT3 & 5.10 & -0.36 & 17.68 & 2.95 & 8.50 & 3.24 & 5.78 & 4.31 & 3.98 & 2.89 & 5.80 & 2.01 \\
\hline AMT4 & 7.95 & -0.49 & 21.35 & 3.24 & 5.45 & 3.36 & 5.98 & 9.05 & 3.64 & 5.19 & 4.79 & 2.39 \\
\hline AMT5 & 8.08 & 2.88 & 21.82 & 3.71 & 2.66 & 5.46 & 6.92 & 9.28 & 2.75 & 4.25 & 5.35 & 2.56 \\
\hline AMT6 & 10.75 & 5.01 & 22.41 & 3.55 & 2.21 & 4.47 & 5.91 & 9.98 & 3.28 & 4.47 & 5.75 & 2.63 \\
\hline CPT1 & 5.63 & 4.14 & 15.34 & 5.61 & 3.78 & 7.61 & -2.63 & 7.00 & 3.19 & 5.17 & 7.55 & 4.03 \\
\hline$\overline{\mathrm{CPT} 2}$ & 3.98 & 10.10 & 12.74 & 7.04 & 7.37 & 10.28 & -9.26 & -2.76 & 3.42 & 5.66 & 6.86 & 5.74 \\
\hline CPT3 & 18.58 & 28.22 & 19.57 & 7.20 & 5.92 & 8.47 & 3.98 & 12.31 & 15.95 & 5.00 & 7.35 & 4.41 \\
\hline CPT4 & 29.62 & 36.24 & 28.04 & 8.36 & 8.02 & 7.11 & 16.20 & 23.71 & 25.82 & 4.58 & 5.79 & 3.57 \\
\hline CPT5 & 40.52 & 47.64 & 40.05 & 8.71 & 6.67 & 4.70 & 31.65 & 32.08 & 33.61 & 5.23 & 7.46 & 4.99 \\
\hline$\overline{\text { CPT6 }}$ & 45.36 & 54.15 & 49.15 & 7.15 & 6.22 & 4.24 & 38.10 & 39.48 & 39.24 & 6.85 & 7.55 & 5.25 \\
\hline RP1 & 44.70 & 50.71 & 49.64 & 6.61 & 5.76 & 5.98 & 35.92 & 40.74 & 35.55 & 4.57 & 7.75 & 6.34 \\
\hline RP2 & 43.03 & 43.70 & 46.51 & 7.48 & 6.69 & 6.26 & 35.18 & 43.70 & 36.82 & 5.86 & 7.85 & 6.26 \\
\hline RP3 & 35.78 & 38.82 & 41.28 & 6.13 & 6.70 & 6.77 & 31.30 & 33.81 & 33.41 & 4.95 & 6.94 & 6.57 \\
\hline RP4 & 30.66 & 29.77 & 32.61 & 6.63 & 6.02 & 4.00 & 26.18 & 26.68 & 26.41 & 4.76 & 6.68 & 4.66 \\
\hline RP5 & 25.05 & 24.68 & 24.78 & 6.41 & 5.04 & 2.77 & 24.11 & 20.12 & 21.92 & 4.78 & 5.82 & 4.89 \\
\hline RP6 & 22.99 & 20.92 & 20.12 & 5.56 & 4.09 & 2.23 & 20.73 & 18.22 & 17.99 & 4.70 & 5.20 & 4.58 \\
\hline RP7 & 21.68 & 19.58 & 19.90 & 5.15 & 3.96 & 3.03 & 16.51 & 21.51 & 18.14 & 3.77 & 5.38 & 4.31 \\
\hline RP8 & 19.99 & 16.77 & 21.42 & 5.33 & 3.83 & 2.28 & 15.97 & 19.44 & 17.05 & 4.62 & 5.35 & 2.91 \\
\hline RP9 & 18.95 & 14.82 & 23.78 & 4.52 & 3.25 & 2.61 & 15.17 & 17.75 & 18.78 & 4.82 & 5.95 & 3.62 \\
\hline RP10 & 15.32 & 15.12 & 20.72 & 3.46 & 3.71 & 3.11 & 13.72 & 18.22 & 20.75 & 5.11 & 6.80 & 3.90 \\
\hline RP11 & 15.72 & 16.94 & 17.67 & 3.20 & 5.49 & 3.23 & 15.27 & 18.48 & 22.31 & 4.22 & 6.66 & 4.34 \\
\hline RP12 & 17.25 & 17.55 & 19.34 & 3.29 & 3.98 & 3.38 & 17.28 & 18.84 & 21.79 & 4.70 & 6.35 & 4.66 \\
\hline RP13 & 17.35 & 15.34 & 23.24 & 3.92 & 3.46 & 3.27 & 15.42 & 22.60 & 17.48 & 4.96 & 6.25 & 4.65 \\
\hline RP14 & 15.36 & 16.77 & 20.18 & 3.67 & 5.27 & 3.30 & 14.40 & 18.04 & 15.75 & 5.45 & 5.50 & 3.33 \\
\hline RP15 & 15.06 & 18.07 & 22.38 & 3.85 & 3.84 & 2.66 & 9.80 & 20.72 & 15.35 & 5.23 & 6.03 & 3.03 \\
\hline RP16 & 16.48 & 16.54 & 18.65 & 2.71 & 3.50 & 3.63 & 12.42 & 23.12 & 17.28 & 4.57 & 7.01 & 3.83 \\
\hline RP17 & 18.10 & 17.70 & 17.67 & 3.75 & 2.96 & 3.49 & 13.92 & 19.35 & 18.24 & 4.87 & 5.63 & 3.76 \\
\hline RP18 & 18.33 & 16.40 & 15.70 & 3.04 & 1.96 & 4.23 & 14.43 & 16.14 & 19.36 & 3.91 & 6.94 & 3.10 \\
\hline
\end{tabular}

High-response group

Mean blood pressure

SEM

Mean blood pressure

SEM

Caffeine L-theanine Placebo Caffeine L-theanine Placebo Caffeine L-theanine Placebo Caffeine L-theanine Placebo

\begin{tabular}{llllllllllllll}
\hline Base & 0 & 0 & 0 & 0 & 0 & 0 & 0 & 0 & 0 & 0 & 0 & 0 \\
\hline AMT1 & 4.96 & 6.00 & 11.74 & 3.66 & 2.30 & 1.97 & 7.41 & 5.87 & 7.14 & 2.65 & 3.20 & 1.62 \\
\hline AMT2 & 4.47 & 5.34 & 12.30 & 2.53 & 1.31 & 3.56 & 2.38 & 1.46 & 4.14 & 2.61 & 2.98 & 2.08 \\
\hline AMT3 & 5.09 & 0.61 & 12.60 & 1.98 & 3.27 & 2.91 & 3.51 & 3.00 & 4.39 & 2.71 & 3.27 & 2.27 \\
\hline AMT4 & 5.73 & 2.14 & 17.07 & 2.39 & 2.20 & 2.83 & 4.22 & 5.31 & 5.39 & 3.34 & 3.22 & 2.00 \\
\hline AMT5 & 5.81 & 5.67 & 16.10 & 2.27 & 3.43 & 4.83 & 5.39 & 4.99 & 4.51 & 2.92 & 3.03 & 1.88 \\
\hline AMT6 & 7.19 & 6.64 & 16.90 & 2.37 & 3.08 & 3.73 & 3.95 & 6.66 & 4.64 & 2.98 & 3.03 & 1.73 \\
\hline CPT1 & 7.70 & 7.64 & 18.30 & 3.54 & 3.66 & 4.12 & 3.16 & 10.21 & 7.52 & 4.15 & 3.32 & 2.35 \\
\hline CPT2 & 8.56 & 9.58 & 16.31 & 3.87 & 3.60 & 5.78 & 0.89 & 1.36 & 6.49 & 4.13 & 3.14 & 2.88 \\
\hline
\end{tabular}


Table 1 Changes in systolic blood pressure and diastolic blood pressure (Continued)

\begin{tabular}{|c|c|c|c|c|c|c|c|c|c|c|c|c|}
\hline CPT3 & 20.21 & 22.98 & 22.82 & 4.57 & 4.24 & 3.62 & 9.71 & 11.34 & 16.82 & 3.16 & 3.73 & 1.73 \\
\hline CPT4 & 28.40 & 30.20 & 29.32 & 5.15 & 6.15 & 1.49 & 18.34 & 20.10 & 23.91 & 2.86 & 2.84 & 2.14 \\
\hline CPT5 & 36.06 & 38.60 & 38.72 & 5.17 & 5.60 & 2.20 & 24.56 & 26.83 & 28.84 & 2.47 & 3.38 & 1.91 \\
\hline CPT6 & 39.39 & 42.83 & 43.18 & 4.30 & 4.62 & 3.75 & 27.31 & 30.29 & 32.04 & 2.90 & 3.73 & 2.23 \\
\hline RP1 & 35.79 & 38.60 & 42.20 & 5.27 & 4.67 & 4.80 & 24.09 & 27.87 & 27.85 & 2.71 & 3.48 & 2.61 \\
\hline RP2 & 32.09 & 31.23 & 36.65 & 6.64 & 5.16 & 4.47 & 19.93 & 25.49 & 24.41 & 3.35 & 3.74 & 2.22 \\
\hline RP3 & 25.97 & 26.03 & 31.75 & 5.68 & 5.50 & 4.00 & 16.69 & 18.37 & 19.62 & 3.04 & 3.84 & 3.18 \\
\hline RP4 & 21.84 & 21.50 & 25.34 & 5.36 & 5.65 & 2.92 & 13.60 & 15.34 & 16.02 & 3.16 & 3.82 & 2.84 \\
\hline RP5 & 18.16 & 17.67 & 19.81 & 5.31 & 4.85 & 2.57 & 12.40 & 12.10 & 12.68 & 3.04 & 3.10 & 2.99 \\
\hline RP6 & 16.11 & 15.78 & 16.97 & 4.49 & 4.38 & 1.68 & 10.79 & 11.10 & 11.09 & 3.20 & 2.71 & 2.72 \\
\hline RP7 & 14.83 & 14.55 & 15.90 & 3.73 & 4.37 & 1.58 & 8.04 & 12.81 & 11.44 & 2.59 & 2.66 & 2.59 \\
\hline RP8 & 13.71 & 12.40 & 16.55 & 3.80 & 3.67 & 1.98 & 8.26 & 10.90 & 10.98 & 3.29 & 2.75 & 1.79 \\
\hline RP9 & 12.59 & 12.25 & 18.04 & 3.83 & 3.38 & 2.17 & 6.76 & 10.14 & 12.08 & 3.11 & 2.99 & 2.36 \\
\hline RP10 & 9.06 & 12.00 & 15.18 & 2.98 & 3.86 & 2.63 & 5.95 & 10.23 & 12.16 & 3.47 & 2.99 & 2.64 \\
\hline RP11 & 10.23 & 12.78 & 13.37 & 2.58 & 3.72 & 2.73 & 7.45 & 10.24 & 12.82 & 2.42 & 2.62 & 2.38 \\
\hline RP12 & 10.90 & 12.94 & 14.80 & 2.89 & 3.04 & 2.93 & 7.04 & 10.61 & 12.76 & 3.19 & 3.18 & 2.62 \\
\hline RP13 & 10.04 & 11.61 & 16.50 & 3.04 & 2.74 & 2.47 & 6.49 & 12.07 & 9.99 & 2.90 & 2.95 & 2.94 \\
\hline RP14 & 8.90 & 12.14 & 13.94 & 2.90 & 3.26 & 2.53 & 5.72 & 8.90 & 9.75 & 3.19 & 3.04 & 1.67 \\
\hline RP15 & 9.30 & 12.54 & 16.81 & 3.12 & 2.76 & 1.97 & 4.38 & 11.49 & 8.88 & 3.33 & 2.78 & 1.70 \\
\hline RP16 & 8.99 & 10.44 & 13.04 & 2.50 & 1.71 & 2.86 & 5.96 & 11.91 & 9.58 & 2.28 & 2.87 & 1.76 \\
\hline RP17 & 9.30 & 11.80 & 12.77 & 2.69 & 2.30 & 3.38 & 5.79 & 9.37 & 10.08 & 3.29 & 2.70 & 2.12 \\
\hline RP18 & 9.66 & 12.08 & 10.03 & 2.33 & 2.53 & 3.43 & 6.17 & 8.93 & 11.50 & 2.43 & 3.06 & 2.57 \\
\hline
\end{tabular}

repeated-measures ANOVA with group (high-response group and low- response group), treatment (L-theanine, caffeine, and placebo), and epoch (six epochs for AMT, CPT and 18 epochs for CPT). Repeated-measures ANOVA with group and treatment was also applied to the task performance data. A Tukey's honestly significant difference (HSD) post hoc test was applied to data groups with significant main effect $(P<0.05)$. Differences in POMS and VAS scores were analyzed using the nonparametric Friedman test to detect differences in treatments. The Wilcoxon signed rank test was further carried out to evaluate the changes among treatments.

\section{Results}

\section{Systolic blood pressure}

Changes in systolic blood pressure and diastolic blood pressure are summarized in Table 1. In the AMT period, there was an interaction effect between treatment and group $(F(2,24)=3.438, \quad P=0.049)$. The high-response group revealed main effects of treatment significantly at AMT4, AMT5, and AMT6 and showed a trend at AMT3 $(\mathrm{F}(2,12)=6.958,5.500,7.195$, and 2.994, $P=0.010$, $0.020,0.009,0.088)$. As shown in Figure 2, the results of Tukey's LSD showed that in the 1-min measurement of the high-response group after the mental tasks, the Ltheanine intake condition tended to decrease the systolic blood pressure in the AMT3 period $(P=0.082)$, and showed a significant effect of lower value in the AMT4, AMT5, and AMT6 periods compared with that of the placebo intake condition $(P=0.008,0.019,0.008)$. Caffeine intake showed a trend of lower systolic blood pressure than the placebo condition only at AMT4, AMT5, and AMT6 ( $P=0.099,0.090,0.068)$.

In the rest periods, systolic blood pressure did not differ significantly among treatments.

No treatment effect was found in the low-response group (Figure 3).

\section{Diastolic blood pressure}

Diastolic blood pressure in the AMT period revealed trends for the main effect of treatment $(F(2,24)=2.577$, $P=0.097)$ and of group $(\mathrm{F}(1,12)=3.361, P=0.092)$. In the high-response group, treatment affected diastolic blood pressure at AMT4 and AMT6 $(\mathrm{F}(2,12)=7.932,4.300$, $P=0.006,0.039)$, and lower values were obtained by $\mathrm{L}$ theanine $(P=0.006,0.056)$ or caffeine intake $(P=0.033$, 0.071) than in the placebo condition. Diastolic blood pressure did not differ significantly among the treatments in the other periods

No treatment effect was found in the low-response group in any of the periods of the blood pressure measurements. 


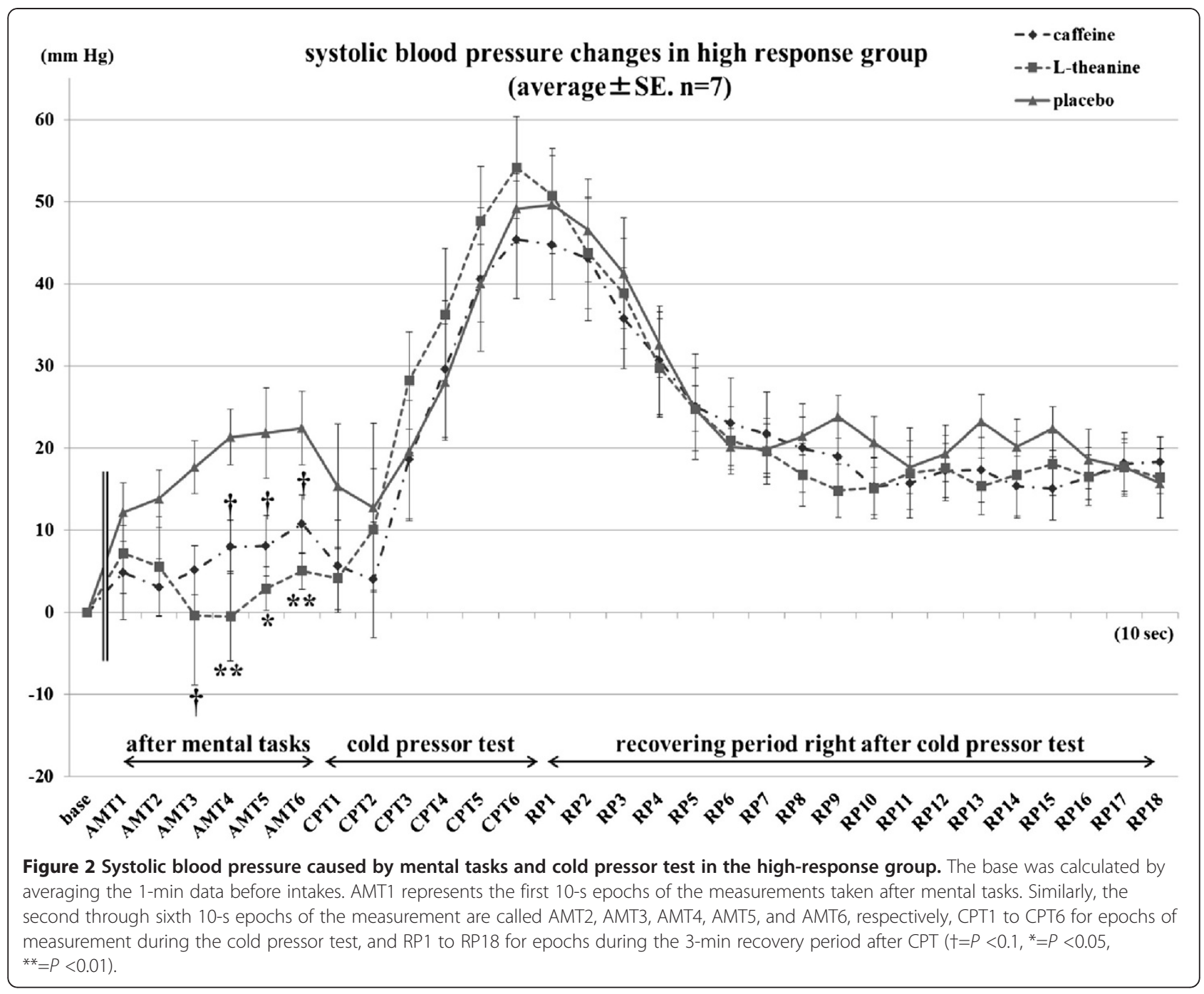

\section{Skin temperature}

Skin temperature was not affected by the different sample intakes in each group or two groups together in this study (data not shown).

\section{POMS and VAS}

Figure 4 presents the significant results of POMS scores. T-A scores and A-H scores showed treatment effects over the two groups together $\left(X^{2}=6.000,6.048, P=0.050\right.$, $0.049)$, and L-theanine intake decreased T-A score below that in the placebo condition $(P=0.004)$.

No difference was obtained among treatments in each group or two groups together for VAS assessments.

\section{Task performance}

There was no interaction effect between group and treatment. Over two groups together, treatment tended to affect the number of answers in $\operatorname{AT}(F(2,26)=3.261$, $P=0.054$ ), and participants answered more questions after caffeine intake than after placebo intake $(P=0.052)$. There was no effect on the accuracy of the answers.

\section{Discussion}

Oral administration of L-theanine significantly changed both systolic and diastolic blood pressures in the highresponse group during the latter part of AMT compared with the placebo condition. These results demonstrated the possibility that L-theanine can attenuate blood pressure elevation induced by mental tasks. This finding agreed with the blood pressure-reducing effect of Ltheanine intake reported in another study, in which theanine inhibited the blood-pressure increase resulting from caffeine intake [15]. The AMT measurements were carried out after participants finished all of the mental tasks and just before the CPT. That is to say, the participants felt stressed not only by the mental tasks but also by, or even more by, their knowledge that their CPT would be taken in a minute. The high-response group included participants who showed large increases in 


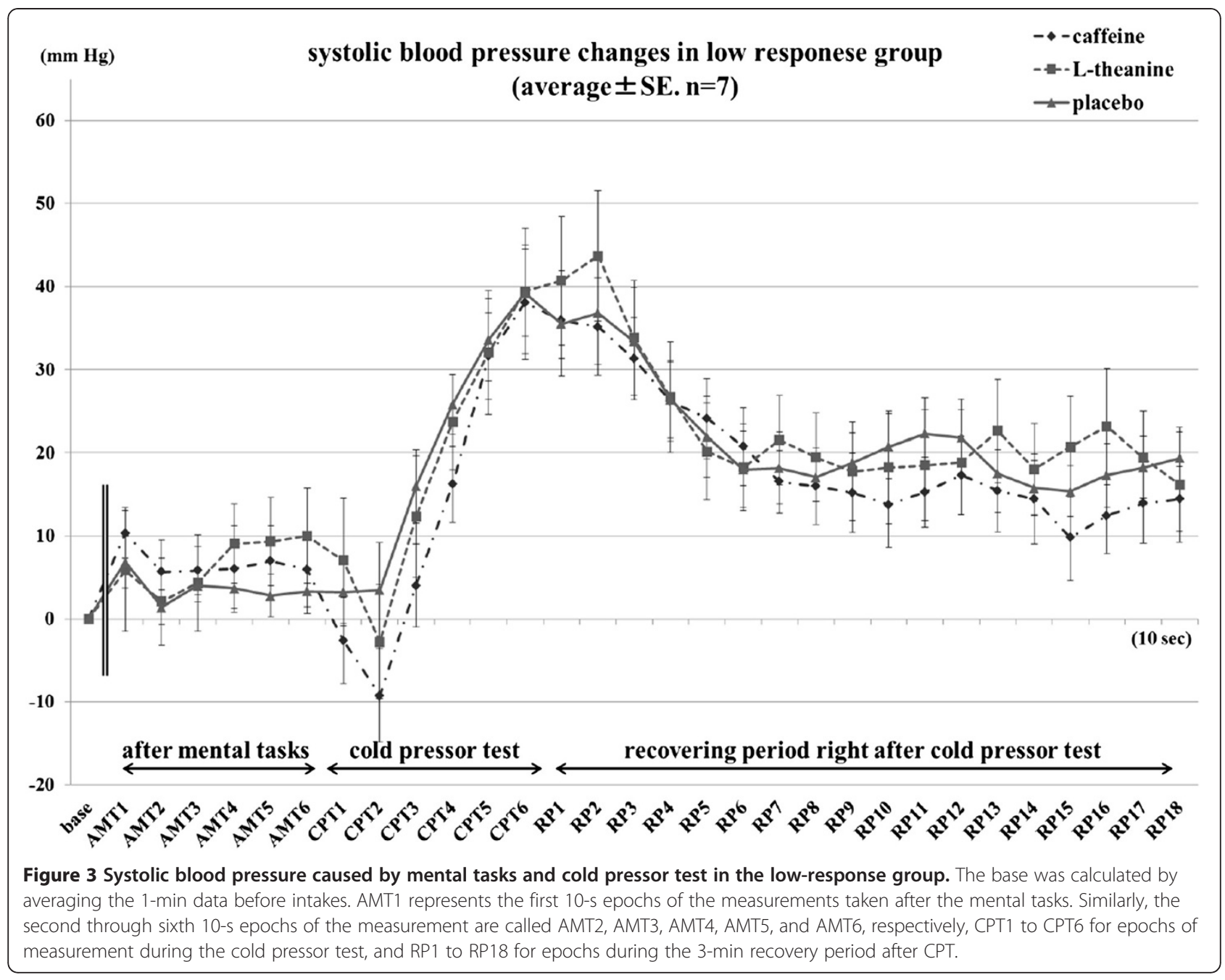

mean systolic blood pressure after the mental tasks in the placebo intake condition, and the range of elevation was 9.46 to $33.88 \mathrm{mmHg}$. It has been considered that young adults who show a large blood-pressure response to psychological stress may be at risk for hypertension as they approach mid-life [9]. From this point of view, participants in the high-response group in this study might be at risk of hypertension. The result showed that the intake of $200 \mathrm{mg}$ L-theanine significantly attenuated the blood pressure response caused by psychological stress in the high-response group. This indicated that Ltheanine reduced blood pressure not only for spontaneously hypertensive rats $[6,7]$ but also for humans at risk of hypertension, despite the lower dose of L-theanine (200/62.8=3.2 $\mathrm{mg} / \mathrm{kg}$ body weight) for the high-response group comparing with $2,000 \mathrm{mg} / \mathrm{kg}$ for hypertensive rats. The mechanism underlying this result might be the same as that reported in Kimura et al. (2007), that Ltheanine could cause anti-stress effects by inhibiting cortical neuron excitation, which attenuates the sympathetic nervous activation response to the acute stress task [5].
Stress may not directly cause hypertension, but it can lead to repeated blood pressure elevations, which can eventually lead to hypertension [8]. With this in mind, L-theanine might be useful for preventing the development of hypertension. Although we could not obtain results of this anti-stress effect from the VAS assessment, the results of POMS scores in T-A indicated that L-theanine intake improved participants' mood by lowering the tension and anxiety caused by psychological stress. This supported the relaxing effect reported in Juneja et al. (1999) that L-theanine can promote the generation of alpha brain waves and induce a relaxed state in humans approximately $40 \mathrm{~min}$ after intake [3].

Contrary to our hypothesis, caffeine also tended to inhibit blood-pressure elevation in this study, and it did not show opposite effects to L-theanine on blood pressure raised by psychological stress. Suleman and Siddiqui (1997 to 2004) suggested that caffeine raised blood pressure during stress by elevating the resting baseline from which the response was measured and not by potentiating the acute blood pressure stress response [12]. The 


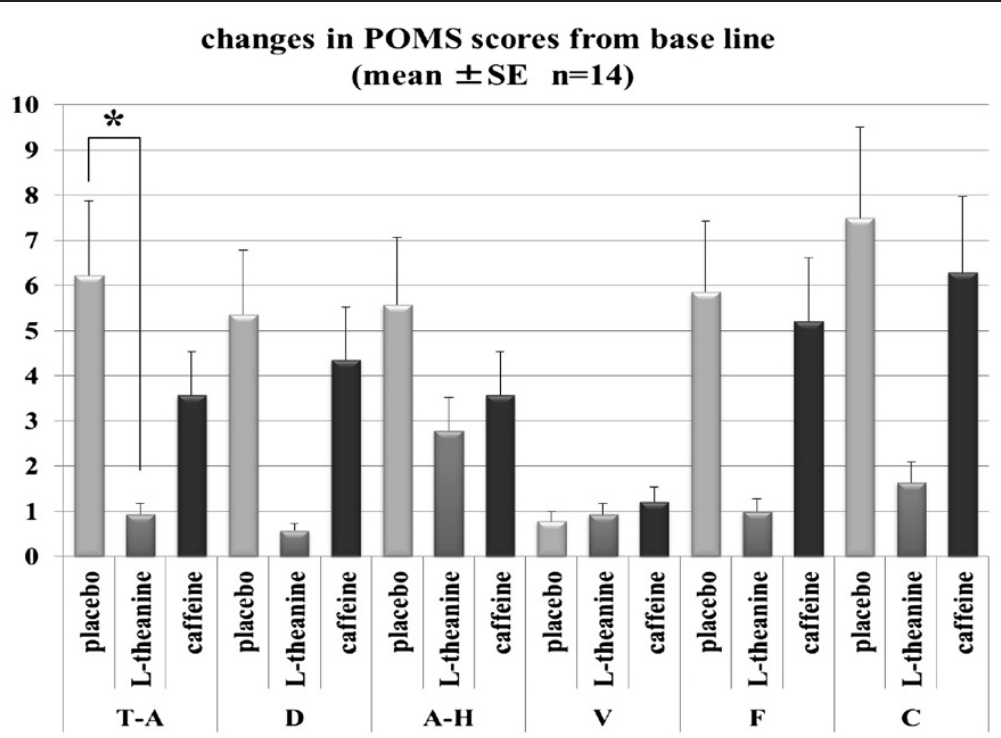

Figure 4 Changes in POMS scores from the baseline after mental tasks. A-H, Anger-Hostility; C, Confusion-Bewilderment; D, Depression-Dejection; F, Fatigue-Inertia; T-A, Tension-Anxiety; V, Vigor-Activity.

psychological stress load used in the current study started right after the sample intake without resting period, which might have been strong and thus potentiated the stress response to a level higher than the response potentiated by caffeine intake. Moreover, Lane and Williams (1987) reported that caffeine potentiated stress-related increases in forearm vasodilation [21]. This might also lower the raised blood pressure measured from the thumb of the left hand in our study.

On the other hand, neither L-theanine nor caffeine decreased the rise in blood pressure caused by CPT compared with the placebo. This might be attributable to the difference in the mechanism between blood pressure elevation by psychological stress and that by the physical stress of pain. Further studies are needed to confirm this and to investigate how L-theanine or caffeine influences the autonomic nervous system responses under other kinds of physical stress.

At last, due to the limitation on the amount of female participants in this study, the possible effects of their menstrual period are difficult to be discussed this time. Thus, there is a possibility that the results might be different if the number of participants is large enough to sort them into four groups: two male groups (the highand the low-response groups) and two female groups (also the high- and low-response groups). We would like to confirm this with larger numbers of both male and female participants in future.

\section{Conclusions}

Our results suggested that L-theanine not only reduces anxiety but also attenuates the rise in blood pressure in high-stress-response adults. In addition, neither Ltheanine nor caffeine showed any effect on decreasing the rise in blood pressure caused by strong physical stress, such as the CPT used in this study.

\section{Abbreviations}

A-H: Anger-hostility; AMT: Measurement after mental tasks; ANOVA: Analysis of variance; AT: Arithmetic mental task; C: Confusion-bewilderment; CPT: Cold pressor test; D: Depression-dejection; DT: Auditory oddball target detection task; F: Fatigue-inertia; HSD: Tukey's honestly significant difference; POMS: Profile of Mood States; T-A: Tension-anxiety; V: Vigor-activity; VAS: Visual analogue scales.

\section{Competing interests}

The authors declare that they have no competing interests.

\section{Authors' contributions}

AY conceived and designed the study, performed the experiments and the statistical analysis, and drafted the manuscript. MM and SM helped to carry out the experiments and to perform data analysis. HY conceived of the study, participated in its design and coordination, and helped to draft the manuscript. All authors have read and approved the final manuscript.

\section{Acknowledgements}

This work was supported in part by grants from the Collaboration of Regional Entities for the Advancement of Technological Excellence (CREATE), research funds provided by the Japan Society and Technology Agency (JST), and by a Grant-in-Aid for Scientific Research (B) provided by the Japan Society for the Promotion of Sciences (JSPS) from the Ministry of Education, Culture, Sports, Science, and Technology of Japan.

Received: 27 September 2012 Accepted: 8 October 2012

Published: 29 October 2012

\section{References}

1. Yokogoshi H, Kobayashi M, Mochizuki M, Terashima T: Effect of theanine, Y-glutamylethylamide, on brain monoamines and striatal dopamine release in conscious rats. Neurochem Res 1998, 23:667-673.

2. Yamada T, Terashima T, Okubo T, Juneja LR, Yokogoshi H: Effects of theanine, r-glutamylethylamide, on neurotransmitter release and its 
relationship with glutamic acid neurotransmission. Nutr Neurosci 2005 8:219-226.

3. Juneja $L R$, Chu DC, Okubo T, Nagato Y, Yokogoshi H: L-theanine-a unique amino acid of green tea and its relaxation effect in humans. Trends Food Sci Technol 1999, 10:199-204.

4. Gomez-Ramirez M, Higgins BA, Rycroft JA, Owen GN, Mahoney J, Shpaner $M$, Foxe JJ: The deployment of intersensory selective attention: a high-density electrical mapping study of the effects of theanine. Clin Neuropharmacol 2007, 30:25-38.

5. Kimura K, Ozeki M, Juneja LR, Ohira H: L-theanine reduces psychological and physiological stress responses. Biol Psychol 2007, 74:39-45.

6. Yokogoshi H, Kato Y, Sagesaka Y, Matsuura T, Kakuda T, Takeuchi N: Reduction effect of theanine on blood pressure and brain 5-hydroxyindoles in spontaneously hypertensive rats. Biosci Biotechnol Biochem 1995, 59:615-618.

7. Yokogoshi $\mathrm{H}$, Kobayashi M: Hypotensive effect of $\mathrm{\gamma}$-glutamylethylamide in spontaneously hypertensive rats. Life Sci 1998, 62:1065-1068.

8. Kulkarni S, O'Farrell I, Erasi M, Kochar MS: Stress and hypertension. Wis Med J 1998, 97:34-38.

9. Matthews KA, Katholi CR, McCreath H, Whooley MA, Williams DR, Zhu S, Markovitz JH: Blood pressure reactivity to psychological stress predicts hypertension in the CARDIA study. Circulation 2004, 110:74-78.

10. Smith HJ, Rogers PJ: Effects of low doses of caffeine on cognitive performance, mood and thirst in low and higher caffeine consumers. Psychopharmacology 2000, 152:167-173.

11. Pelligrino DA, Xu HL, Vetri F: Caffeine and the control of cerebral hemodynamics. J Alzheimers Dis 2010, Suppl 1:S51-S62.

12. Suleman A, Siddiqui NH: Haemodynamic and cardiovascular effects of caffeine. Pharmacy. Int J Pharm, http://www.priory.com/pharmol/caffeine. $\mathrm{htm}$.

13. Owen GN, Parnell H, Bruin EA, Rycroft JA: The combined effects of L-theanine and caffeine on cognitive performance and mood. Nutr Neurosci 2008, 11:193-198.

14. Haskell CF, Kennedy DO, Milne AL, Wesnes KA, Scholey AB: The effects of L-theanine, caffeine and their combination on cognition and mood. Biol Psychol 2008, 77:113-122.

15. Rogers PJ, Smith JE, Heatherley SV, Pleydell-Pearce CW: Time for tea: mood, blood pressure and cognitive performance effects of caffeine and theanine administered alone and together. Psychopharmacology (Berl) 2008, 195:569-577.

16. Yokogosh H, Mochizuki M, Saitoh K: Theanine-induced reduction of brain serotonin concentration in rats. Biosci Biotechnol Biochem 1998, 62:816-817.

17. Van der Pijl PC, Chen L, Mulder TPJ: Human disposition of I-theanine in tea or aqueous solution. J Funct Foods 2010, 2:239-244.

18. Terashima T, Takido J, Yokogoshi H: Time-dependent changes of amino acids in the serum, liver, brain and urine of rats administered with theanine. Biosci Biotechnol Biochem 1999, 63:615-618.

19. Fredholm BB, Bättig K, Holmén J, Nehlig A, Zvartau EE: Actions of caffeine in the brain with special reference to factors that contribute to its widespread use. Pharmacol Rev 1999, 51:83-133.

20. Shibahara N, Matsuda H, Umeno K, Shimada Y, Itoh T, Terasawa K: The responses of skin blood flow, mean arterial pressure and R-R interval induced by cold stimulation with cold wind and ice water. J Auton Nerv Syst 1996, 61:109-115.

21. Lane JD, Williams RB Jr: Cardiovascular effects of caffeine and stress in regular coffee drinkers. Psychophysiology 1987, 24:157-164.

doi:10.1186/1880-6805-31-28

Cite this article as: Yoto et al:: Effects of L-theanine or caffeine intake on changes in blood pressure under physical and psychological stresses. Journal of Physiological Anthropology 2012 31:28.

\section{Submit your next manuscript to BioMed Central and take full advantage of:}

- Convenient online submission

- Thorough peer review

- No space constraints or color figure charges

- Immediate publication on acceptance

- Inclusion in PubMed, CAS, Scopus and Google Scholar

- Research which is freely available for redistribution

Submit your manuscript at www.biomedcentral.com/submit
Biomed Central 